\title{
Soliton Radiation Beat Analysis of Optical Pulses Generated from Two CW Lasers
}

\author{
M. Zajnulina ${ }^{\text {a) }}$, M. Böhm ${ }^{\text {b) }}$ K. Blow ${ }^{\text {c) }}$, A. A. Rieznik ${ }^{\text {d) }}$, D. Giannone ${ }^{\text {a) }}$, R. Haynes ${ }^{\text {a) }}$, M. M. Roth ${ }^{\text {a) }}$ \\ ${ }^{a}$ innoFSPEC-VKS, Leibniz Institute for Astrophysics, An der Sternwarte 16, 14482 Potsdam, Germany \\ ${ }^{b}$ innoFSPEC-InFaSe, University of Potsdam, Am Mühlenberg 3, 14476 Golm, Germany \\ ${ }^{c}$ Aston Institute of Photonic Technologies, Aston Triangle, Birmingham, B4 7ET, United Kingdom \\ ${ }^{d}$ Instituto Tecnologico de Buenos Aires and CONICET, Buenos Aires, Argentina
}

We propose a fibre-based approach for generation of optical frequency combs (OFC) with the aim of calibration of astronomical spectrograph in the low and medium-resolution range. This approach is based on two steps: in the first step, an appropriate state is generated and subsequently moulded in the second step into the desired OFC. In the system we propose, the first step is realised by injection of two continuous-wave (CW) lasers into a conditional single-mode fibre, whereas the second step generates a broad OFC by using the optical solitons generated in step one as initial condition. We investigate the conversion of a bichromatic input wave produced by two initial CW lasers into a train of optical solitons which happens in the fibre used in step one. Especially, we are interested in the soliton content of the pulses created in this fibre. For that, we study different initial conditions (a single cosine-hump, an Akhmediev breather, and a deeply-modulated bichromatic wave) by means of Soliton Radiation Beat Analysis and compare the results to draw conclusion about the soliton content of state generated during the first step.

Optical frequency combs constitute a discrete optical spectrum with lines that are equidistantly positioned. Frequency combs generated in modelocked lasers have been proposed and already successfully tested as calibration sources for high-resolution astronomical spectrographs. However, there is a variety of novel astronomical instruments that operate in the low- and medium resolution range. They also would profit from the deployment of frequency combs as calibration marks. We propose a fibre-based approach for optical frequency comb generation that is specifically suitable for spectrographs with low and medium resolution. This approach consists of two fibres fed with two continuous lasers. To be able to generate broadband, stable, and low-noise frequency combs, we need to understand the optical pulse formation in different fibre stages. In this paper, we focus our attention on the pulse build-up in the first fibre stage of the proposed approach and study it numerically by means of the Soliton Radiation Beat Analysis.

\section{INTRODUCTION}

Optical frequency combs (OFC) constitute an array of equidistantly-spaced spectral lines that have nearly equal intensity over a broad spectral range $e^{1,2}$. Combs generated in mode-locked lasers have been successfully demonstrated as calibration sources for astronomical spectrographs deployed for high-resolution spectroscopy ${ }^{3-9}$. In this resolution range, the comb line spacing typically varies from $1 \mathrm{GHz}$ to $25 \mathrm{GHz}^{10,11}$. Such novel instruments like PMAS, MUSE, 4MOST, a future Multiobject-Instrument for the E-ELT (ELT-MOS) operate, however, in the low- and medium resolution range and need OFC having line spacings going from slightly below $100 \mathrm{GHz}$ to a few hundreds of $\mathrm{GHz}^{12-14}$.

We propose an all optical fibre-based approach for generation of OFC in the low- and medium resolution range with tuneable line spacing. It consists of two fibre stages where the first stage is a conventional single-mode fibre, the second one is an Erbium-doped fibre. The fibres get pumped by two continuous-wave lasers (CW) generating a bichromatic deeply modulated cosinewave as the input field ${ }^{15-17}$.

The goal of this study is to understand and to control the temporal shape of the first fibre stage output which is important, because this temporal profile will define the pulse and, thus, the OFC build-up in the second fibre stage. To be able to generate broadband $\mathrm{OFC}$, one should reduce the pulse duration in the second fibre stage as much as possible. Further, a perfectly periodic output after the first stage is needed to achieve a high level of the OFC lines sharpness. To fulfil this requirement, it is necessary to start with a periodic initial condition, e.g. a bichromatic cosinusoidal optical field with a proper laser frequency separation (LFS) as we have in the proposed fibre-based approach.

An effective pulse compression in the time domain is realised if any interfering optical solitons are exited in the first fibre stage. Additionally, the usage of solitons is helpful for stabilising the output structure of this stage. However, if too many solitons are excited they will tend to break up and impact the periodicity of the temporal shape. The propagation behaviour of a single soliton is well known ${ }^{18}$. However, we are here interested in a state when many solitons strongly overlap with each other. The soliton overlap that occurs in our system it not sufficiently understood yet. To get insight into the nonlinear dynamics that take place in the first fibre stage, we apply the soliton radiation beat 
analysis (SRBA) that will help us to retrieve the soliton content ${ }^{19}$. Our system is described by a nonintegrable propagation equation. The SRBA, however, is capable of dealing with nonintegrable equations with arbitrary initial conditions ${ }^{20}$.

To decode the SRBA spectra, we analyse different initial conditions. More precise, additionally to the desired bichromatic cosinusoidal input field, we also study a single cosine-hump since this will provide us with the behaviour when single solitons are well separated. To study the contrary case of strongly overlapping solitons, we decode the case of a maximally compressed Akmediev breather as initial condition.

This paper is structured as follows: in Sec. 2, we present the experimental setup for generation of OFCs in fibres and the according mathematical model, the concept of SRBA is depicted in Sec. 3 and the result are shown in Sec. 4 , a conclusion is drawn in Sec. 5.

\section{EXPERIMENTAL SETUP AND MATHEMATICAL MODEL}

The schematic presentation of the experimental setup for generation of OFC in the low- and medium resolution range for the purpose of the spectrograph calibration is shown in Fig. $1^{15-}$ 17. The generation of an OFC starts with two independent and free-running $\mathrm{CW}$ lasers that have equal intensity and feature relative frequency stability of $10^{-8}$ sufficient for astronomical applications in the medium-resolution range. Laser 1 is fixed at the angular frequency $\omega_{1}$, Laser 2 has tunable frequency $\omega_{2}$, the resulting modulated cosine-wave has the frequency $\omega_{c}=$ $\left(\omega_{1}+\omega_{2}\right) / 2$ that coincides with the central wavelength at $1531 \mathrm{~nm}$. The initial laser frequency separation, $\quad L F S=\left|\omega_{2}-\omega_{1}\right| / 2 \pi, \quad$ is $L S F=$ 78.125 GHz. Fiber A is a conventional single-mode fiber, B is an Erbium-doped fiber. In fiber A, a sequence of temporally periodic soliton-like structures with widths in ps-range evolves out of the initial deeply modulated cosine-wave having a narrowband OFC as spectrum. In fiber $B$, the soliton-like pulses are compressed to the fs-range and the OFC broadened. Pulse compression in an amplifying medium can be considered as an

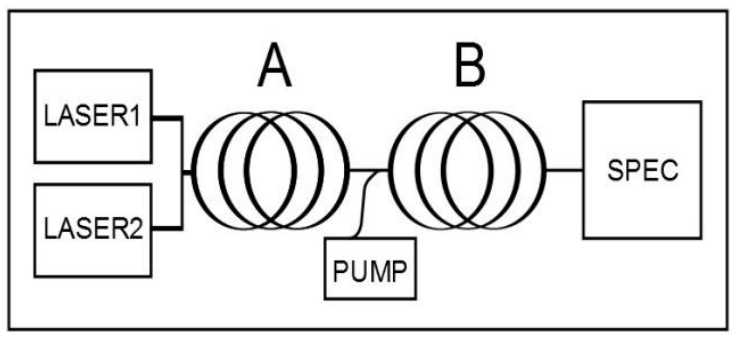

FIG. 1. Schematic presentation of the motivating setup. LASER1: fixed CW laser, LASER2: tunable CW laser, A: conventional single-mode fiber, B: Er-doped fiber, PUMP: pump laser for fiber B, SPEC: astronomical spectrograph alternative technique to the compression in dispersion-decreasing fibers ${ }^{21-23}$.

We model the light propagation in fibre A by means of the generalised nonlinear Schrödinger equation (GNLS) for a slowly varying optical field envelope $A=A(z, t)$ in the co-moving frame ${ }^{22,24,25}$ :

$$
\begin{aligned}
\frac{\partial A}{\partial z}=i \sum_{k=2}^{3} \frac{i^{k}}{k !} \beta_{k} \frac{\partial^{k} A}{\partial t^{k}} & +i \gamma\left(1+\frac{i}{\omega_{0}} \frac{\partial}{\partial t}\right) \times \\
& \times\left(A \int_{-\infty}^{\infty} R\left(t^{\prime}\right)\left|A\left(t-t^{\prime}\right)\right|^{2} d t^{\prime}\right)
\end{aligned}
$$

where $\beta_{k}=\left(\frac{\partial^{k} \beta}{\partial \omega^{k}}\right)_{\omega=\omega_{0}}$ is the value of the dispersion order at the carrier frequency $\omega_{0}$, whereas $\gamma=\frac{\omega_{0} n_{2}}{c \cdot s}$ defines the nonlinear coefficient with $n_{2}$ being the nonlinear refractive index of silica, $S$ the effective mode area, and $c$ the speed of light. The delayed Raman effect is incorporated via $h_{R}(t)$ into the response function that includes both, the electronic contribution assumed to be nearly instantaneous and the contribution set by vibration of silica molecules and reads as:

$$
R(t)=\left(1-f_{R}\right) \delta(t)+f_{R} h_{R}(t)
$$

with $f_{R}=0.245$ denoting the fraction of the delayed Raman response to the total nonlinear polarisation. The function $h_{R}(t)$ is defined as:

$$
\begin{aligned}
& h_{R}(t)=\left(1-f_{b}\right) h_{a}(t)+f_{b} h_{b}(t), \\
& h_{a}(t)=\frac{\tau_{1}^{2}+\tau_{2}^{2}}{\tau_{1} \tau_{2}^{2}} \exp \left(-\frac{t}{\tau_{2}}\right) \sin \left(\frac{t}{\tau_{1}}\right), \\
& h_{b}(t)=\left(\frac{2 \tau_{b}-t}{\tau_{b}^{2}}\right) \exp \left(-\frac{t}{\tau_{b}}\right)
\end{aligned}
$$

where $\tau_{1}=12.2 \mathrm{fs}$ and $\tau_{2}=32 \mathrm{fs}$ are the characteristic times of the Raman response of silica and $f_{b}=0.21$ represents the according vibrational instability with $\tau_{b} \approx 96 \mathrm{fs}^{24,25}$.

For fibre A, following parameters are chosen: $\quad \beta_{2}=-15 \frac{\mathrm{ps}^{2}}{\mathrm{~km}}, \beta_{3}=0.1 \frac{\mathrm{ps}^{3}}{\mathrm{~km}}, \quad$ and $\gamma=$ $2 \mathrm{~W}^{-1} \mathrm{~km}^{-1}$. The numerical solution of Eq. 1 is performed within a temporal window of $128 \mathrm{ps}$ using the interaction picture method in combination with the local error method with $2^{14}$ sample points ${ }^{26}$.

The propagation of light in fibre A with a subsequent SRBA is calculated using three different types of initial condition (IC):

1. A single cosine-hump that is expected to evolve into a soliton with a sech-profile due to the properties of Eq. 1 (FIG. 2(A) $)^{18}$ :

$$
A(z=0, t)= \begin{cases}0, & |t|>6.4 \text { ps } \\ N \sqrt{P_{0}} \cos \left(\omega_{c} t\right), & |t| \leq 6.4 \text { ps }\end{cases}
$$

2. A maximally compressed Akhmediev breather (FIG. 2(B)) ${ }^{27,28}$ : 
A

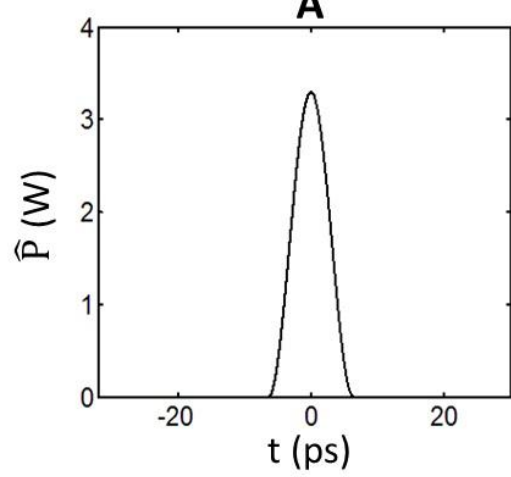

B

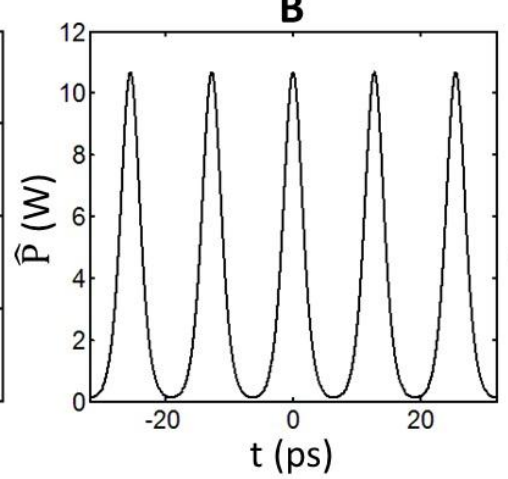

C

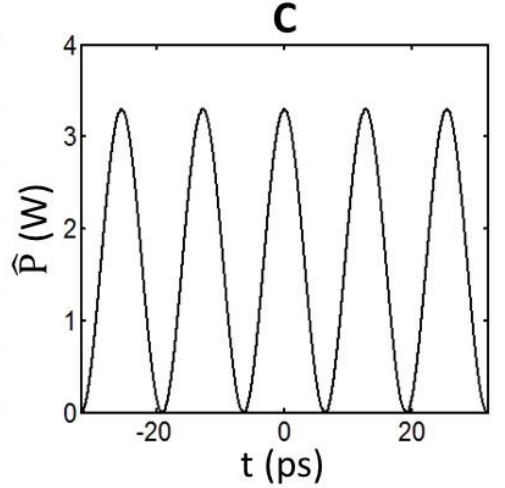

FIG. 2. Example of three types of initial conditions with initial power of $P_{0}=3.3 \mathrm{~W}$ : A. Single cosine-hump, B. Maximally compressed Akhmediev breather, C. Deeply modulated cosine-wave according to the proposed setup

$$
A(z=0, t)=N \sqrt{P_{0}} \frac{(1-4 a)+\sqrt{2 a} \cos \left(\omega_{\bmod } t\right)}{\sqrt{2 a} \cos \left(\omega_{\bmod } t\right)-1}
$$

with the coefficient $a$ that is defined as $a=\frac{1}{2}\left(1-\frac{\omega_{\text {mod }}^{2}\left|\beta_{2}\right|}{4 \gamma P_{0}}\right)$ and the modulational frequency $\omega_{\text {mod }}=4 \pi L F S$.

3. Deeply modulated cosine-wave that mimics the starting condition of the motivating setup (FIG. 2(C) $)^{15-17}$ :

$A(z=0, t)=N \sqrt{P_{0}} \cos \left(\omega_{c} t\right)$.

To increase the resemblance between the conditions presented in Eq. 5 and Eq. 6, we choose $a=0.08$. In Eq. 4, Eq. 5, and Eq. 6, $N$ is the scale soliton order given by

$$
N^{2}=\frac{\gamma P_{0}}{(2 \pi \cdot L F S)^{2}\left|\beta_{2}\right|} .
$$

\section{SOLITON RADIATION BEAT ANALYSIS}

The SRBA will hepl us to determine the content of optical solitons generated in our system for different initial conditions (Eq. 4, Eq. 5, and Eq. 6). It will provide us with information about solitons' structure and order ${ }^{19,20}$.

We explain the numerical technique of SRBA by taking the example when propagation of the initial wave (Eq. 6) occurs for the input power of $P_{0}=3.3 \mathrm{~W}$. The total fibre length is $L=20 \mathrm{~km}$. Generally, the resolution of the power spectrum

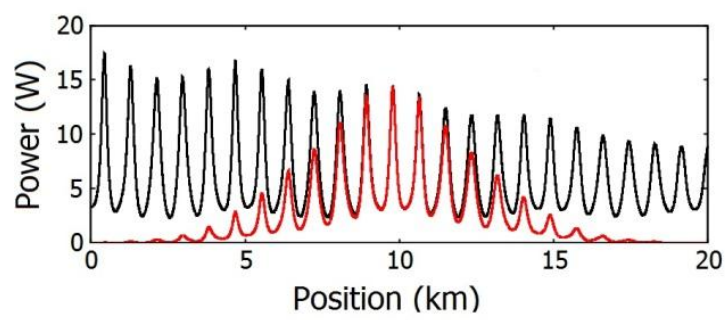

FIG. 3. Optical power at $t=0$ (black) and apodized optical power at $t=0$ (red) vs. propagation distance for $P_{0}=3.3 \mathrm{~W}$ plots within the SRBA strongly depends on the total fibre length chosen for simulation. Precisely, it goes with $1 / L$ with $L$ being the total propagation length. Therefore, it is advisable to choose long fibre lengths to obtain high-quality graphs.

To perform the SRBA, one first needs to calculate the optical field along the propagation distance, i.e. $A(z, t)$, then the optical power $P(z, t)=|A(z, t)|^{2}$. After that, optical power at $t=0$ gets extracted, i.e. $P(z)=P(z, t=0)$. As presented in Fig. 3, $P(z)$ oscillates over the propagation distance (black curve) ${ }^{29}$. This oscillation contains information about the solitons involved and manifests itself in strong peaks in the power spectrum $\tilde{P}(Z)$ obtained via a Fourier transform of $P(z)$ (Fig. 4, black curve). To emphasize the spectral power peaks, we perform the Fourier transform on the apodised data that are depicted as red curve in Fig. 3. The resulting spectral power is shown as red curve in Fig. 4. The apodisation function is a Gaussian with the maximum positioned at the middle of the total propagation distance:

$$
f(k)=\exp \left[-\left(\frac{k-K / 2}{b K}\right)^{2}\right]
$$

with $1 / b$ being the apodization strength and $k \in[0, \ldots, K]$, where $\mathrm{K}=20000$ is the total number of distance sampling points. For further studies, $b$ is set to $b=0.2$

\section{RESULTS}

Now, using the SRBA technique presented in

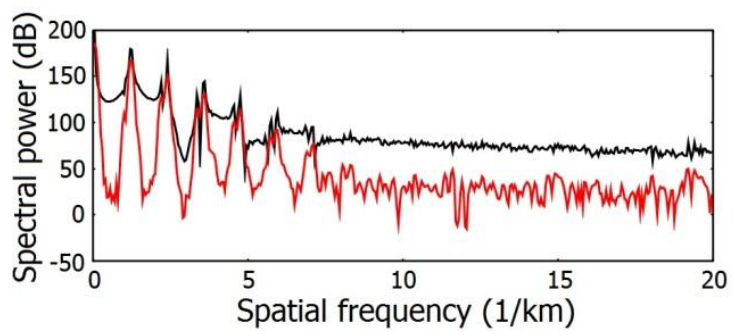

FIG. 4. Spectral power of non-apodized optical power (black) and apodized optical power (red) vs. spatial frequency (cf. FIG. 3) 
Sec. 3, we analyse our system for input power values of $0.03 \mathrm{~W} \leq P_{0} \leq 6.00 \mathrm{~W}$, the total length of fibre A is chosen to be $L=20 \mathrm{~km}$. We plot the spectral power as a function of spacial frequency $Z$ and the input power $P_{0}$. In all graphs presented below, one will see a strong peak for any values of $P_{0}$ and $Z=0 \mathrm{~km}^{-1}$. This peak arises during the Fourier transform from the optical into frequency domain and corresponds to the average value of the optical power $P(z)$. Since it does not contain any pieces of information about the soliton content, we will exclude it from consideration. Another feature of the SRBA is the appearance of the overtones of the oscillations. The overtones give us no further information about the soliton content. Therefore, they will be also excluded from consideration.

\subsection{Single cosine-hump as initial condition}

Fig. 4 shows the power spectrum obtained using a single cosine-hump as IC (Eq. 1 and Eq. 4). It is typical for single solitons to arise at a positive threshold value of the input power, $P_{0}>0 \mathrm{~W}$ and to evolve depending on ${\sqrt{P_{0}}}^{18,19}$. In our case, such typical behaviour is presented by branch $\mathrm{S} 1$ that starts from $P_{0}=0.7 \mathrm{~W}$ at $Z=0 \mathrm{~km}^{-1}$. Thus, we can conclude that $\mathrm{S} 1$ constitutes a beating of a single soliton with the background. The scale order of this soliton is $N=0.62$ according to Eq. 7. For the Nonlinear Schrödinger Equation without additional terms (NLS), the threshold for creation of fundamental solitons is $N=0.5^{18}$. Thus, $\mathrm{S} 1$ can be identified as a fundamental soliton.

The next soliton branch $\mathrm{S} 2$ arises at $P_{0}=$ $3.3 \mathrm{~W}$ and $Z=0 \mathrm{~km}^{-1}$ and has the scale soliton order $N=1.35$. For the NLS, the threshold for a second-order soliton is at $N=1.5^{18}$. Since the scale order of the soliton involved into the branch S2 is below this threshold, $\mathrm{S} 2$ can be identified as another fundamental soliton. The energy growth of S1 with increasing input power starts decreasing as soon as S2 appears. This has a change of the slope of $\mathrm{S} 1$ as a result meaning that the energy provided by the initial field is now split between both solitons.
The branches named as $\mathrm{O} 1, \mathrm{O} 2, \mathrm{O} 3$ are the overtones of $\mathrm{S} 1$.

\subsection{Akhmediev breather as initial condition}

Fig. 6 represents the power spectrum obtained by choosing a maximally compressed Akhmediev breather as IC (Eq. 1 and Eq. 5). Apparently, there are three input-power dependent regions with different soliton behavior, i.e. $0.03 \mathrm{~W} \leq P_{0}<0.54 \mathrm{~W}, \quad 0.54 \mathrm{~W}<P_{0}<2.3 \mathrm{~W}$, and $P_{0}>2.3 \mathrm{~W}$.

For input powers $0.03 \mathrm{~W} \leq P_{0}<0.54 \mathrm{~W}$, we observe three branches $\mathrm{C} 1, \mathrm{C} 2, \mathrm{C} 3$ that are not well resolved. To obtain a more detailed presentation of $\mathrm{C} 1, \mathrm{C} 2$, and $\mathrm{C} 3$, we choose a fibre length of $L=50 \mathrm{~km}$. The resulting power spectrum is depicted in Fig. 7. The theree branches and their overtones are now presented in more detail. Obviously, there is no energetic thereshold for the formation of the $\mathrm{C}$-branches visible, i.e. $\mathrm{C} 1, \mathrm{C} 2$, and $\mathrm{C} 3$ arise for $P_{0}=0 \mathrm{~W}$ at $Z=0.3 \mathrm{~km}^{-1}$, $Z=1.2 \mathrm{~km}^{-1}$, and $Z=2.6 \mathrm{~km}^{-1}$, respectively. As discussed in Sec 4.1, a single cosine-hump as initial condition does not have enough energy to form a soliton at low input powers. However, as we have here a periodic initial condition, a collective soliton state can be formed even with less input power, creating the $\mathrm{C}$-branches. The required energy is provided by the initial condition that incorporates $\cos$-functions that have infinite energy for $t \rightarrow \pm \infty$ (Eq. 5). By analogy to an electronic state in a crystal, the C-branches can be referred to as a collective soliton crystal state (cf. Ref. 30).

For input powers $P_{0}>0.54 \mathrm{~W}$, significant groups of branches arise out of C1, C2, C3. Branch M1(A) originates from C1, M1(B) from C2, and M3 from C3. Branches M1(A) and M1(B) merge for increasing value of $P_{0}$. To explain this behaviour, we start at the higher-energetic edge of this region, i.e. at $P_{0}=2.3 \mathrm{~W}$. Beyond this region, i.e. $P_{0}>2.3 \mathrm{~W}$ the soliton branches (S1 and $\left.\mathrm{S} 2\right)$ are temporally well separated since their duration is small compared to their temporal separation (cf. Ref. 31, Ref. 32). As the input power and so the

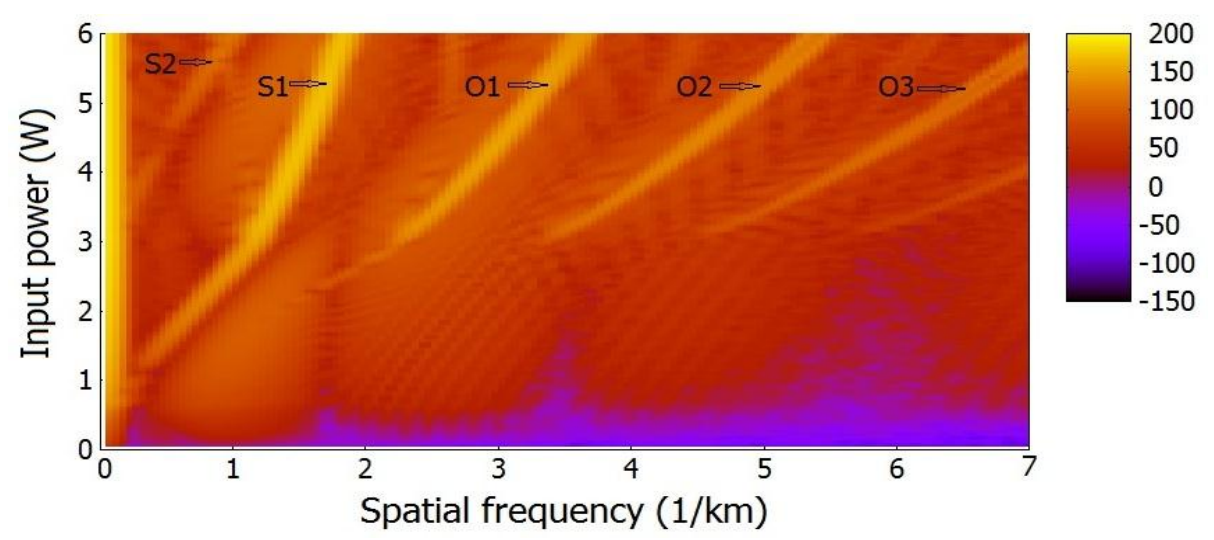

FIG. 5. Power spectrum for a single cosine-hump as initial condition 


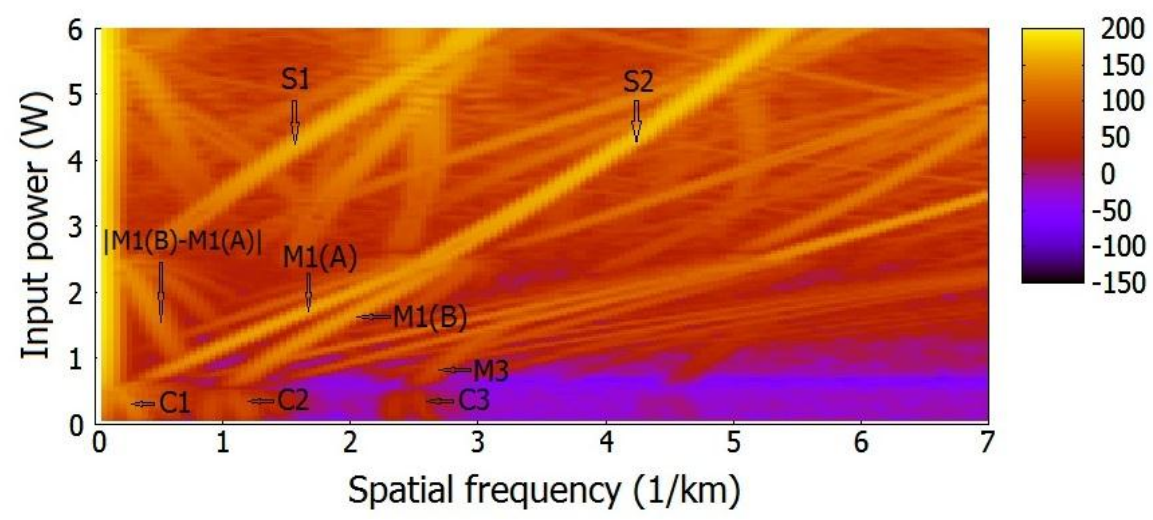

FIG. 6. Power spectrum for an Akhmediev breather as initial condition

solitons' energies decrease in the region $P_{0}<$ $2.3 \mathrm{~W}$, the duration of solitons increases. Eventually, the solitons overlap in the temporal domain which makes solitons' energies split: M1(A) and M1(B) arise ${ }^{18}$. In analogy to the energy splitting in molecules, M1(A) and M1(B) can be regarded as a common soliton molecule state $\mathrm{M}{ }^{33-}$ 38

The branch $|\mathrm{M} 1(\mathrm{~B})-\mathrm{M} 1(\mathrm{~B})|$ constitutes the mixing frequency between $\mathrm{M} 1(\mathrm{~A})$ and $\mathrm{M} 1(\mathrm{~B})$.

For exactly $P_{0}=0.54 \mathrm{~W}$, we observe a perfect oscillation-free Akhmediev breather which manifests inself in a thin line that parts the collective soliton state from the state of soliton molecules in Fig. 7.

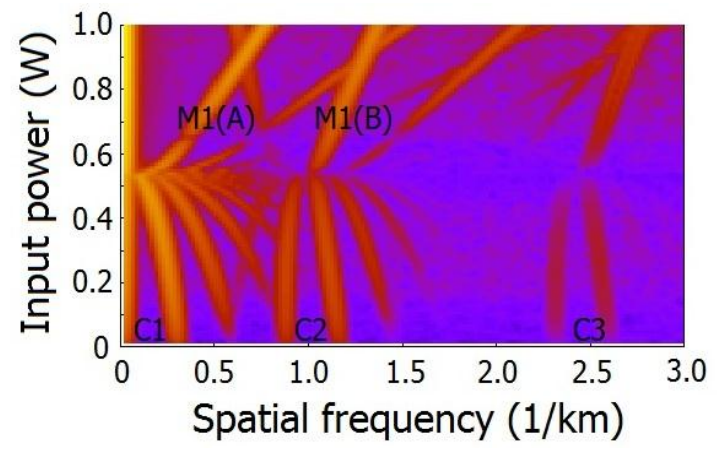

FIG. 7. Power spectrum for the Akhmediev initial condition at low input powers

\subsection{Modulated cosine-wave as initial condition}

Fig. 8 presents the power spectrum obtained using a deeply modulated cosine-wave as IC (Eq. 6). The most prominent soliton branch $\mathrm{S} 1$ starts at $P_{0}=0 \mathrm{~W}$ and $Z=0.65 \mathrm{~km}^{-1}$. Similar to the case when Akhmediev breather was chosen as IC, the branch S1 has no energetic threshold constituing a collective soliton crystal state. The input power range where the collective state exists is $0 \mathrm{~W} \leq P_{0}<1.3 \mathrm{~W}$. In case of an Akhmediev breather as IC, the region of the collective state was separated from the molecule state at $P_{0}=0.54 \mathrm{~W}$. Here, the transition from the soliton crystal to a state of well separated solitons occurs continuously which is indicated by a smooth evolution of the S1branch for increasing input powers. The molecule state is even missing.
At $P_{0}=3.3 \mathrm{~W}$ and $Z=0 \mathrm{~km}^{-1}$, a second soliton branch S2 arises. Obviously, it shows the behavior of a single soliton beating with the background (cf. Sec. 4.1). According to Eq. 7, it has the soliton scale order of $N=1.35$ meaning that $\mathrm{S} 2$ represents a fundamental soliton.

The overtones of $\mathrm{S} 1$ arise at $P_{0}=0 \mathrm{~W}$ and $Z=1.2 \mathrm{~km}^{-1}(\mathrm{O} 1)$ and $Z=1.8 \mathrm{~km}^{-1}(\mathrm{O} 2)$. The branches $|\mathrm{S} 1-\mathrm{S} 2|,|\mathrm{S} 1+\mathrm{S} 2|,|\mathrm{O} 1-\mathrm{S} 2|$, and $|01+\mathrm{S} 2|$ constitute the frequencies emerged from the beating of solitons with each other or of solitons and the overtones.

\section{CONCLUSION}

The aim of this study was to understand the build-up of the temporal pulse shape in the first fibre stage of the proposed setup for generation of optical frequency combs for the calibration of astronomical spectrographs in the low- and medium resolution range. A bichromatic deeply modulated cosine-wave is chosen as optical input field within the framework of this setup. In particular, the temporal behaviour needs to be considered for two limiting cases: for high input powers and input powers that go to zero. The temporal build-up of a bichromatic cosine-wave in the first fibre stage is easily understood if compared with the cases when a single cosine-hump and a maximally compressed Akhmediev breather are chosen as initial condition.

It was expected that a single cosine-hump as initial condition will evolve into a soliton while propagation through the fibre ${ }^{39}$. This behaviour was confirmed in our studies: we observed the emergence of two fundamental solitons depending on the input power, the soliton $\mathrm{S} 1$ emerged for the input power of $0.7 \mathrm{~W}$ and the soliton $\mathrm{S} 2$ for $3.3 \mathrm{~W}$. Comparing the relative soliton energy content at the limit of high input powers, we see that oscillation of the soliton $\mathrm{S} 1$ is faster than the soliton S2, namely, the spatial frequency of $\mathrm{S} 1$ is $1.8 \mathrm{~km}^{-1}$ and the frequency of $S 2$ is $0.9 \mathrm{~km}^{-1}$ for the input power of $6.0 \mathrm{~W}$.

The case when a maximally compressed Akhmediev breather is chosen as initial condition is more complicated as the previous one. Thus, we observe the build-up of an oscillation-free Akhmediev breather at the input power of $0.54 \mathrm{~W}$. 


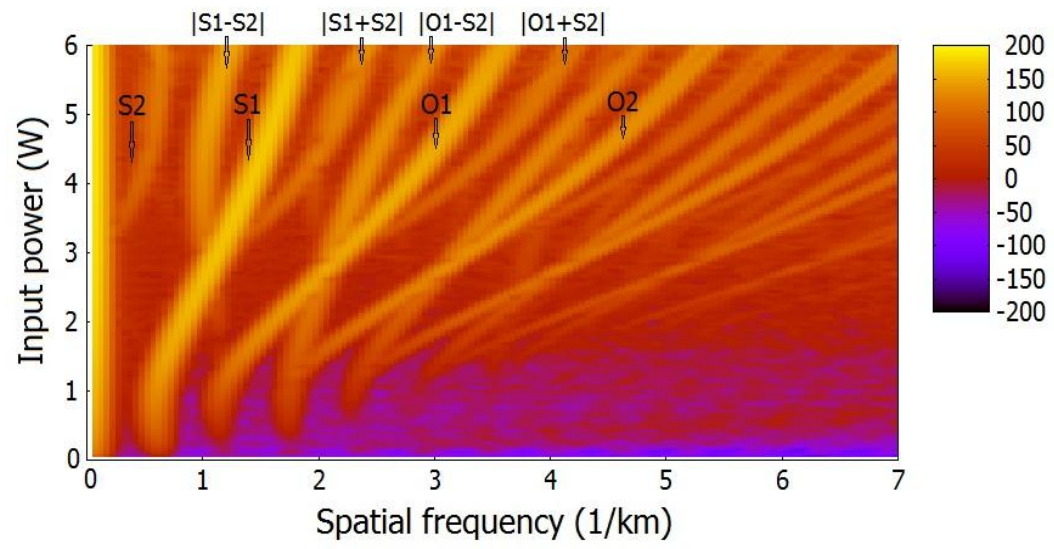

FIG. 8. Power spectrum for a deeply modulated cosine-wave as initial condition

For lower input powers, we observe an oscillating collective soliton crystal state. For input powers $0.54 \mathrm{~W}<P_{0}<2.3 \mathrm{~W}$, there is an intermediate soliton molecule state that is followed by the state of separated solitons as the input power increases. In the high input-power limit we observe we observe two separated solitons, S1 and S2. S1 has less energy than S2 and oscillates slower. So, S1 has the spatial frequency of $3.0 \mathrm{~km}^{-1}$ and $\mathrm{S} 2$ the frequency $5.3 \mathrm{~km}^{-1}$ at the input power of $6.0 \mathrm{~W}$. Besides these two solitons, there are many other oscillations that make the usage of an Akhmediev breather as initial condition in a real setup unsuitable, since these oscillations will affect the quality of optical frequency combs in terms of stability and noise evolution.

The case when a bichromatic deeply modulated cosine-wave is chosen as initial condition according to the proposed setup for optical frequency combs generation is quantitatively and qualitatively similar to the case when we have only a single cosine-hump as initial condition. That means there are two separated solitons S1 and S2 for the input power of $6.0 \mathrm{~W}$, the faster soliton $\mathrm{S} 1$ has the spatial frequency $1.7 \mathrm{~km}^{-1}$, the slower soliton $\mathrm{S} 2$ the frequency $0.6 \mathrm{~km}^{-1}$. In the low input-power limit, the buildup of optical structures is similar to the case when an Akhmediev breather is chosen as initial condition. However, the input power is too low to give rise to a broad optical frequency comb. In the intermediate input-power region $\left(2.0 \mathrm{~W}<P_{0}<\right.$ $3.0 \mathrm{~W}$ ), there is state that is a distinct state featuring nonlinear soliton dynamics. This dynamics is comparably simple and gives rise to formation of a good-natured optical frequency comb. Thus, this input-power region should be chosen in a real experiment.

${ }^{1}$ R. Holzwarth, T. Udem, T. W. Hänsch, Physical Review Letters, Vol. 85, No. 11(2000)

${ }^{2}$ S. T. Cundiff, J. Yen, Reviews of Modern Physics, Vol. 75 (2003)

${ }^{3}$ M. T. Murphy, T. Udem, R. Holzwarth,, A. Sizmann, L. Pasquini, C. Araujo-Hauck, H. Dekker, S. D’Odorico,
M. Fischer, T. W. Hänsch, A. Manescau, Monthly Notices of the Royal Astronomical Society, Vol. 380, No. 2 (2007)

${ }^{4}$ D. A. Braje, M. S. Kirchner, S. Osterman, T. Fortier, S. A. Diddams, European Physical Journal D, Vol. 48, Issue 1 (2008)

${ }^{5}$ T. Wilken, C. Lovis, A. Manescau, T. Steinmetz, L. Pasquini, G. Lo Curto, Proceedings of SPIE, Vol. 7735, 77350T-1 (2010)

${ }^{6}$ H.-P. Doerr, T. J. Kentischer, T. Steinmetz, R. A. Probst, M. Franz, R. Holzwarth, T. Udem, T. W. Hänsch, W. Schmidt, Proceedings of SPIE, Vol. 8450, 84501G (2012)

${ }^{7}$ G. Lo Curto, A. Manescau, G. Avila, L. Pasquini, T. Wilken, T. Steinmetz, R. Holzwarth, R. Probst, T. Udem, T. W. Hänsch, J. I. González Hernández, M. Esposito, R. Rebolo, B. Canto Martins, J. R. de Medeiros, Proceedings of SPIE 8446, 84461W (2012)

${ }^{8}$ T. Steinmetz, T. Wilken, A. Araujo-Hauck, A. Holzwarth, T. W. Hänsch, L. Pasquini, A. Manescau, S. D'Odorico, M. T. Murphy, T. Kentischer, W. Schmidt, T. Udem, frequency Science, Vol. 321, No. 5894 (2008)

${ }^{9}$ K. Griest, Whitmore, J. B., Wolfe, A. M., Prochaska, J. X., J. C. Howk, G. W. Marcy, The Astrophysical Journal, 708:158-170 (2010)

${ }^{10}$ S. Osterman, S. Diddams, M. Beasley, C. Froning, L. Hollberg, P. MacQueen, V. Mbele, A. Weiner, Proceedings of SPIE 6693, 66931G-1 (2007)

${ }^{11}$ S. Osterman, G. G. Ycas，S. A. Diddams，F. Quinlan, S. Mahadevan, L. Ramsey, C. F. Bender, R. Terrien, B. Botzer, S. Sigurdsson, S. L. Redman, Proceedings of SPIE 8450 (2012)

${ }^{12}$ M. M. Roth, A. Kelz, T. Fechner, T. Hahn, S.M. Bauer, T. Becker, P. Böhm, L. Christensen, F. Dionies, J. Paschke, E. Popow, D. Wolter, J. Schmoll, U. Laux, W. Altmann, Publications of the Astronomical Society of the Pacific, Vol. 117, Issue 832 (2005)

${ }^{13}$ A. Kelz, S. M. Bauer, I. Biswas, T. Fechner, T. Hahn, J.-C. Olaya, E. Popow, M. M. Roth, O. Streicher, P. Weilbacher, R. Bacon, F. Laurent, U. Laux, J. L. Lizon, M. Loupias, R. Reiss, G. Rupprecht, Proceedings of SPIE 7735, 773552 (2010)

${ }^{14}$ R. S. de Jong, O. Bellido-Tirado, C. Chiappini, E. Depagne, R. Haynes, et al., Proceedings of SPIE 8446, 84460T (2012)

${ }^{15}$ J. M. Chavez Boggio, A. A. Rieznik, M. Zajnulina, M Böhm, D. Bodenmüller, M. Wysmolek, H. Sayinc, J. 
Neumann, D. Kracht, R. Haynes, M. M. Roth, Proceedings of SPIE 8434 (2012)

${ }^{16}$ M. Zajnulina, J. M. Chavez Boggio, A. A. Rieznik, R. Haynes, M. M. Roth, Proceedings of SPIE 8775 (2013)

${ }^{17}$ M. Zajnulina, M. Böhm, K. Blow, J. M. Chavez Boggio, A. A. Rieznik, R. Haynes, M. M. Roth, Proceedings of SPIE 9151 (2014)

${ }^{18}$ J. R. Taylor, Optical Solitons - Theory and Experiment, (Cambridge University Press, 1992)

${ }^{19}$ M. Böhm, F. Mitschke, Physical Review E 73, 066615 (2006)

${ }^{20}$ M. Böhm, F. Mischke, Applied Physics B 86 (2007)

${ }^{21}$ S. V. Chernikov, E. M. Dianov, Optics Letters Vol. 18, No. 7 (1993)

${ }^{22}$ A. A. Voronin, A. M. Zheltikov, Physical Review A Vol. 78, Issue 6 (2008)

${ }^{23}$ Q. Li, J. N. Kunz, P. K. A. Wai, Journal of Optical Society of America B Vol. 27, No. 11 (2010)

${ }^{24}$ G. P. Agrawal, Nonlinear Fiber Optics (Academic Press, 2012)

${ }^{25}$ G. P. Agrawal, Applications of Nonlinear Fiber Optics (Academic Press, 2008)

${ }^{26} \mathrm{~S}$. Balac, Fernandez, F. Mahe, F. Mehats, R. TexierPicard, HAL 00850518v1 (2013)

${ }^{27}$ J. M. Dudley, G. Genty, F. Dias, B. Kibler, N. Akhmediev, Optics Express Vol. 17, No. 24 (2009)

${ }^{28}$ B. Kibler, J. Fatome, C. Finot, G. Millot, G. Genty, B. Wetzel. N. Akhmediev, F. Dias, J. M. Dudley, Nature Scientific Reports 2, No. 463 (2012)

${ }^{29}$ N. F. Smyth, Optics Communications 175 (2000)

${ }^{30}$ A. Hause, F. Mitschke, Physical Review A 82 (2010)

${ }^{31}$ C. Mahnke, F. Mitschke, Applied Physics B 116, Issue 1 (2014)

${ }^{32}$ C. Mahnke, F. Mitschke, Physical Review A 85 (2012)

${ }^{33}$ S. M. Alamoudi, U. Al Khawaja, B. B. Baizakov, Physical Review A 89 (2014)

${ }^{34}$ A. Boudjemaa, U. Al Khawaja, Physical Review A 88 (2013)

${ }^{35}$ P. Rohrmann, A. Hause, F. Mitschke, Physical Review A 87 (2013)

${ }^{36} \mathrm{P}$. Rohrmann, A. Hause, F. Mitschke, Scientific Reports 2:866 (2012)

${ }^{37}$ A. Hause, H. Hartwig, M. Böhm, F. Mitschke, Physical Review A 78 (2008)

${ }^{38}$ A. Hause, H. Hartwig, B. Seifert, M. Böhm, F. Mitschke, Physical Review A 75 (2007)

${ }^{39}$ H. A. Haus, IEEE Spectrum (1993) 